\title{
Effect of angiotensin receptor blockade on central aortic systolic blood pressure in hypertensive Asians measured using radial tonometry: an open prospective cohort study
}

Hui Hwang Teong ${ }^{1}$, MD, MRCP, Adeline Mei Lin Chin ${ }^{1}$, MD, MRCP, Ashish Anil Sule ${ }^{1}$, MBBS, FRCP, Jam Chin $\underline{T a y}^{1}$, MBBS, FRCP

\begin{abstract}
INTRODUCTION Central aortic systolic pressure (CASP) has been shown to be a stronger predictor of cardiovascular events than brachial blood pressure (BP). Different classes of drugs have differential effects on CASP and brachial BP. This open prospective cohort study aimed to observe changes in CASP (measured using radial tonometry) among hypertensive Asians after 12 weeks of treatment with valsartan, an angiotensin receptor blocker (ARB).

METHODS Patients with treatment-naïve hypertension or uncontrolled hypertension who were on non-ARB therapy were eligible for inclusion. Patients with uncontrolled BP (i.e. $\geq 140 / 90 \mathrm{mmHg}$ ) received valsartan for 12 weeks. The patients' brachial systolic and diastolic BP (SBP and DBP), and CASP changes were monitored using the BPro® watch.

RESULTS The mean age of the 44 enrolled patients was 35 years. At baseline, the mean BP and CASP were 150.2/91.4 $\pm 10.6 / 9.4 \mathrm{mmHg}$ and $136.3 \pm 12.2 \mathrm{mmHg}$, respectively. Valsartan reduced SBP, DBP and CASP by $14.9 \pm 10.7 \mathrm{mmHg}$, $10.9 \pm 8.4 \mathrm{mmHg}$ and $15.3 \pm 10.9 \mathrm{mmHg}$, respectively (all $\mathrm{p}<0.001$ ). Every $1.0-\mathrm{mmHg}$ reduction in brachial SBP resulted in a $0.8-\mathrm{mmHg}$ reduction in CASP $(p<0.001)$. A CASP cut-off of $122.5 \mathrm{mmHg}$ discriminated between controlled and uncontrolled BP (sensitivity $74 \%$, specificity $88 \%$ ).

CONCLUSION Using radial tonometry, we demonstrated good correlation between CASP and brachial SBP reductions after 12 weeks of treatment with valsartan in our study cohort. Correlation analysis between CASP and SBP reductions may be useful for demonstrating whether a drug is able to lower CASP beyond lowering SBP.
\end{abstract}

Keywords: BPro ${ }^{\circledR}$ watch, central aortic systolic blood pressure, radial tonometry, valsartan

\section{INTRODUCTION}

Central aortic systolic pressure (CASP) has been shown to be a stronger predictor of cardiovascular events than brachial blood pressure (BP). ${ }^{(1)}$ CASP, which is the pressure that is 'seen' by the left ventricle, has a more direct effect on end organs than brachial systolic blood pressure (SBP). While it is true that brachial SBP is generally higher than CASP and a relatively high correlation exists between the two, we cannot predict the degree of $\mathrm{BP}$ amplification with accuracy using statistical calculations. ${ }^{(2,3)}$ This is because pulse wave reflection is affected by arterial compliance, and factors such as age, height, heart rate and type of drug used. It has also been reported that different drugs have differential effects on CASP beyond peripheral BP lowering. ${ }^{(4)}$

Angiotensin II is known to play a role in arterial stiffness, which affects wave reflection and, hence, CASP. Some studies conducted in Western populations have shown that angiotensin II receptor blockers (ARBs) have more favourable effects on CASP than other classes of antihypertensive drugs (e.g. beta blockers and diuretics). ${ }^{(5-8)}$

Previous studies have employed the SphygmoCor ${ }^{\circledR}$ device (AtCor Medical Pte Ltd, West Ryde, NSW, Australia) to perform arterial waveform analysis and measure CASP using the generalised transfer function, a recognised noninvasive method of central pressure measurement. The BPro ${ }^{\circ}$ watch (HealthSTATS
Int' Pte Ltd, Singapore) is a more recently patented device capable of reliably capturing radial arterial waveforms at the wrist and measuring CASP using the $\mathrm{N}$-point moving average method. This tonometric method has been validated against the gold standard of direct aortic root measurement during cardiac catheterisation, with excellent correlation $(r=0.99) .{ }^{(9)}$ A recent study involving patients with type I diabetes mellitus also showed that there was good agreement between the CASP readings measured using both the SphygmoCor and BPro devices; ${ }^{(10)}$ i.e. any differences observed were within the acceptable limits recommended by the Association for the Advancement of Medical Instrumentation (AAMI) and the European Society of Hypertension (ESH). Notably, the BPro device is also capable of 24-hour ambulatory BP monitoring (24h ABPM).

The objective of the present study was to: (a) demonstrate the effect of valsartan, an ARB drug, on CASP over 12 weeks in an Asian cohort with uncontrolled hypertension using the BPro watch; and (b) analyse the relationship between brachial and central BP changes.

\section{METHODS}

This was an open prospective cohort study without a parallel comparative or control group. From February 2009 to December 2011, patients who presented at the General Medicine Clinic of

${ }^{1}$ Department of General Medicine, Tan Tock Seng Hospital, Singapore

Correspondence: Dr Hui Hwang Teong, Senior Consultant, Department of General Medicine, Tan Tock Seng Hospital, 11 Jalan Tan Tock Seng, Singapore 308433. hui_hwang_teong@ttsh.com.sg 
Tan Tock Seng Hospital, Singapore, were invited to participate in the study if they were aged $\geq 18$ years and had a BP $\geq 140 / 90 \mathrm{mmHg}$. These patients could be newly diagnosed with hypertension (i.e. treatment-naïve) or had already been diagnosed with hypertension and on non-ARB therapy for $\geq 1$ month. If the patient had diabetes mellitus or chronic kidney disease, a stricter inclusion threshold of BP $\geq 130 / 80 \mathrm{mmHg}$ was applied, based on the treatment goals published in the seventh report of the Joint National Committee on Prevention, Detection, Evaluation and Treatment of High Blood Pressure (JNC 7).

Patients were excluded if they had any of the following characteristics: white coat phenomenon; secondary hypertension; serum creatinine $>150 \mathrm{mmol} / \mathrm{L}$ (based on blood test done within the preceding six months); diabetes mellitus with a history of recurrent heart failure, stroke, or angioplasty within the past three months; acute stroke, myocardial infarction, coronary bypass or unstable angina within the past six months; overt heart failure; valvular heart disease (confirmed on echocardiography); liver cirrhosis; and malignancy within the last five years. Pregnant and lactating women were also excluded, in view of the known teratogenic effects of drugs in the ARB class.

The study was approved by the Domain Specific Research Board of the National Healthcare Group, Singapore, and conducted in accordance with the Declaration of Helsinki and Good Clinical Practice Guidelines. All patients gave written informed consent before being enrolled in the study.

At the baseline visit, the $\mathrm{BP}$ readings on both arms were measured using an oscillometric device (MC3100TM; HealthSTATS Int' Pte Ltd, Singapore) after the patient had sat quietly for five minutes. Three more BP readings were taken on the arm that had the higher reading, at an interval of 30-60 seconds between measurements. If the average of the subsequent three readings showed uncontrolled hypertension (i.e. $\mathrm{BP} \geq 140 / 90 \mathrm{mmHg}$ or $\mathrm{BP} \geq 130 / 80 \mathrm{mmHg}$ for patients with diabetes mellitus or chronic kidney disease), the patient was enrolled into the study. Using the BPro watch, the patient's CASP was measured at the clinic. Thereafter, the patient wore the BPro watch for 24h ABPM. In this study, the sleep time was standardised for all patients from 12 midnight to $6 \mathrm{am}$, according to the settings of the BProSOft ${ }^{\circledR}$ 24-hour ABPM Application Software program (HealthSTATS Int' Pte Ltd, Singapore). The purpose of the $24 \mathrm{~h} \mathrm{ABPM}$ is to identify patients with the white coat effect so that treatment could be withheld from these patients (as is routinely done in clinical practice). Secondly, as CASP is believed to be affected by the white coat phenomenon, ${ }^{(11)}$ it made sense to exclude these patients to improve the accuracy of the results.

The BPro watch is a wrist-bound BP monitor that works by applanation tonometry, with a hemispheric plunger placed on the radial artery. It is capable of sampling radial waveforms at a frequency of $60 \mathrm{~Hz}$; 10 -second blocks of radial waveforms are captured and central haemodynamic indices, including CASP and central pulse pressure, can be calculated from these waveforms using the A-Pulse CASP $®$ Application Software program (HealthSTATS Int' Pte Ltd, Singapore). Other than measuring CASP, the device can also monitor ambulatory BP and make measurements at 15-minute intervals. The accuracy of the BPro watch in measuring $24 \mathrm{~h} \mathrm{ABPM}$ has been validated according to the modified ESH protocol and AAMI standard. ${ }^{(12)}$

Upon return of the device on the following day, the $24 \mathrm{~h}$ ABPM report was downloaded. Patients with abnormal results (i.e. daytime average $\geq 135 / 85 \mathrm{mmHg}$ or $\geq 130 / 80 \mathrm{mmHg}$ if the patient had diabetes mellitus or chronic kidney disease) were started on valsartan (suggested starting dose of OM $80 \mathrm{mg}$ ). If the patient's clinic BP was still not controlled (according to JNC 7 guidelines) by Week 4, the valsartan dose was increased (suggested dose increment of $80 \mathrm{mg}$ ). In essence, the valsartan dose that was titrated at Week 4 would be the final stable dose taken for the remaining eight weeks, until Week 12. Although there was no forced titration of the dose, the investigator could decide on the starting and final doses, depending on his/her clinical assessment of the patient's BP and profile (as would be the case in actual clinical practice). The clinic brachial SBP and diastolic BP (DBP), and clinic CASP of all the treated patients were measured using the BPro set at Weeks 4 and 12 (Fig. 1).

Based on a previous study that examined the effect of valsartan, the difference between pre- and post-treatment CASP levels was estimated to be $10 \mathrm{mmHg}{ }^{(5)}$ The sample size calculated based on an estimated standard deviation (SD) of $20 \mathrm{mmHg}$ was 44 patients; with the use of this sample size, there was a $90 \%$ chance of correctly rejecting the null hypothesis ( $\alpha=0.05$, twotailed). ${ }^{(13)}$ Assuming that $25 \%$ of the recruited patients have normal 24h ABPM (i.e. white coat phenomenon), a total of 60 patients must be enrolled for the study.

Summary statistics were calculated for all data collected. Mean \pm SD was reported for continuous variables, while frequency (percentage) was presented for categorical variables. Paired $t$-test was used to examine reductions in brachial SBP, brachial DBP and CASP, from Weeks 0-12. The data collected at Week 4 was not analysed in detail, as the drug dose was interim and administered for only four weeks. Multiple linear regression was used to assess the impact of gender, treatment history, final dose and baseline SBP on CASP reduction. Correlation analysis and simple linear regression were also performed to determine the relationship between brachial SBP and central SBP reductions. The receiver operating characteristic (ROC) curve and logistic regression were used to identify the optimal CASP cut-off for discriminating between controlled and uncontrolled BP. Brachial SBP was dichotomised into controlled and uncontrolled BP groups using a cut-off of 140/90 mmHg. All data analysis was performed using SPSS Statistics version 17.0 (SPSS Inc, Chicago, IL, USA).

\section{RESULTS}

A total of 60 patients who presented at our hospital from February 2009 to December 2011 were enrolled in the study. Among these 60 patients, seven eventually withdrew from the study for various reasons (rejection of drug treatment or noncompliance to follow-up visits). Of the 53 patients who completed the study, one patient, whose waveform capture was repeatedly unsatisfactory, was eventually excluded from the analysis, i.e. only the results of 52 patients were analysed (Fig. 2). 


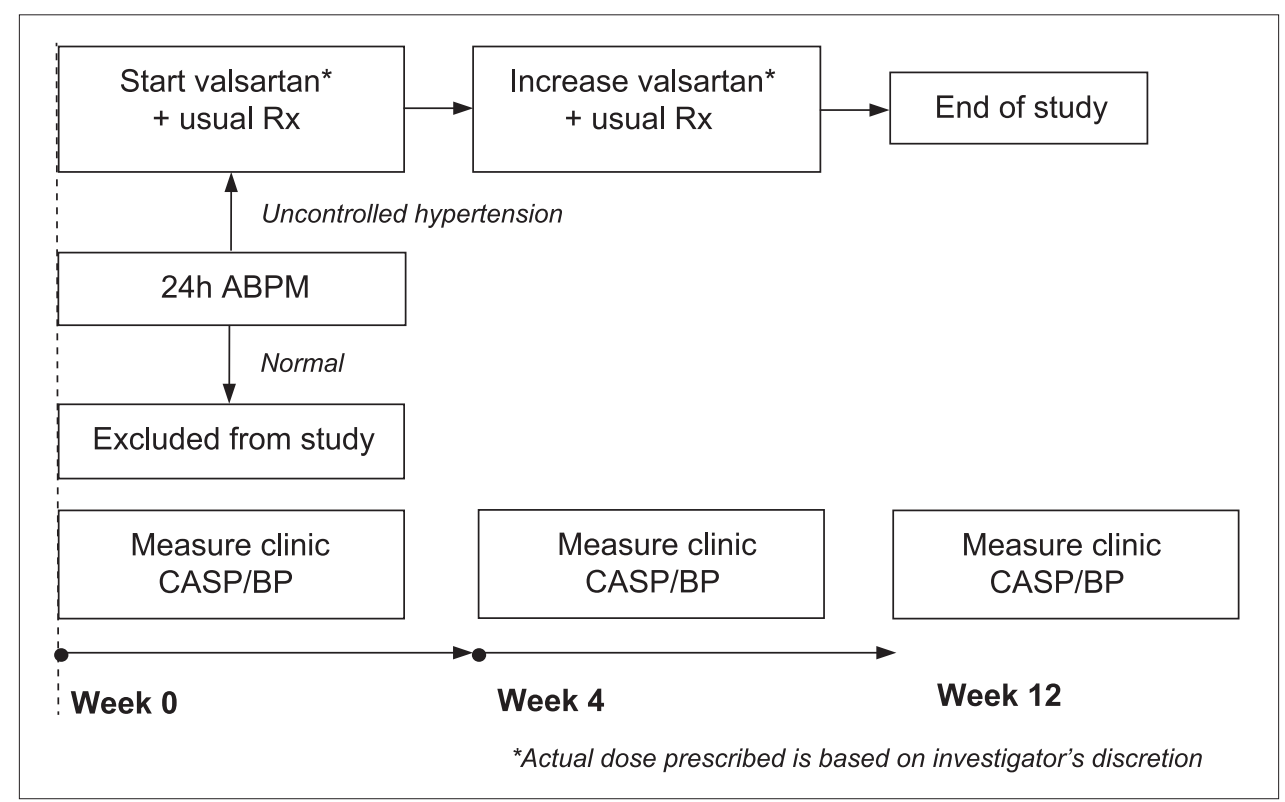

Fig. 1 Flow chart shows the algorithm of the present study. ABPM: ambulatory blood pressure monitoring; BP: blood pressure; CASP: central aortic systolic pressure

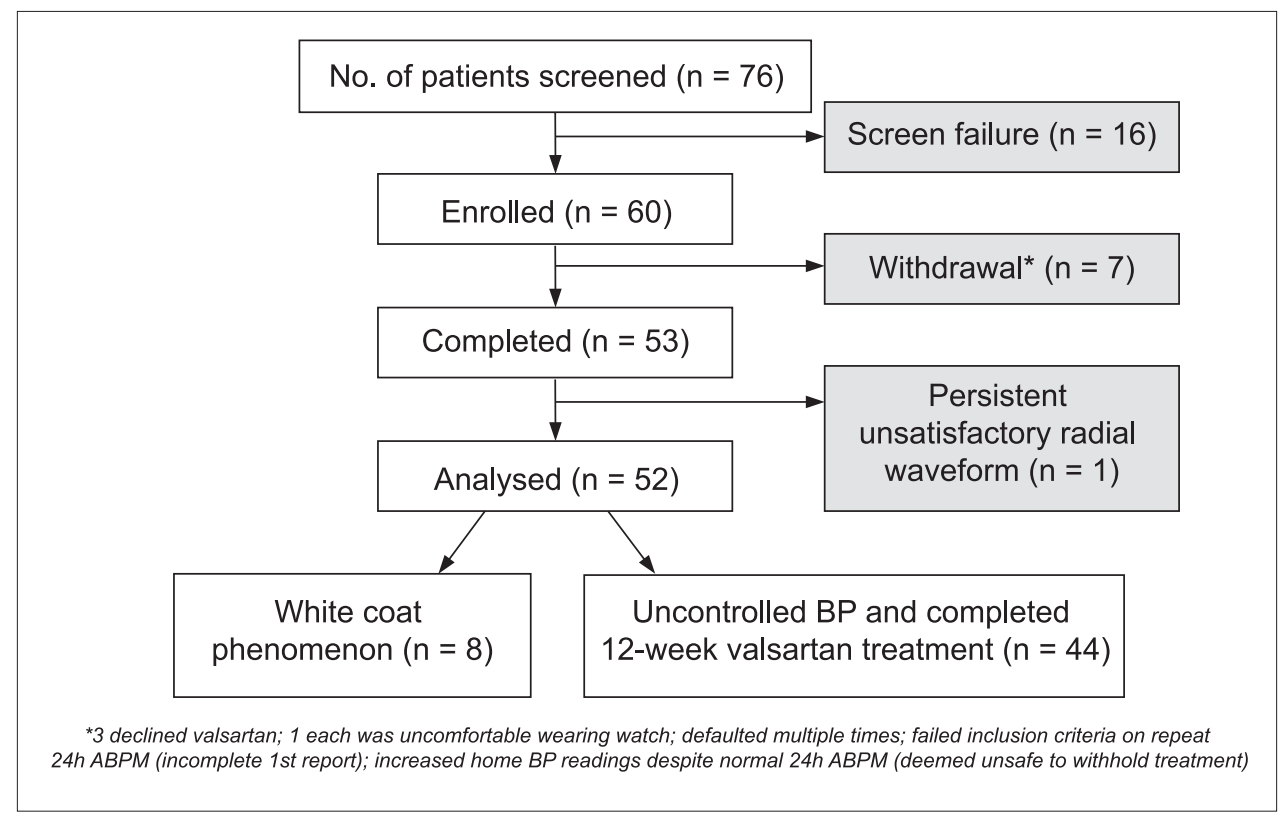

Fig. 2 Flow chart shows the recruitment process of the present study. 24h ABPM: 24-hour ambulatory blood pressure monitoring; BP: blood pressure

Based on the 24h ABPM, 44 (85\%) patients were found to have uncontrolled hypertension and started on valsartan treatment. Table I shows the baseline demographics and characteristics of the treatment cohort. The mean age of the patients was $35 \pm 16$ years, with $75 \%$ male and $80 \%$ Chinese patients. The mean duration of hypertension was 3.5 years and $39 \%$ had at least one other cardiovascular risk factor. Approximately one-third ( $n=14,32 \%$ ) of the patients were on pre-existing treatments, most of whom were on monotherapy and calcium channel blockers. At baseline, three patients were on angiotensin-converting enzyme inhibitors (ACE-I); they were able to tolerate the addition of the study drug (i.e. valsartan). The baseline 24h ABPM daytime average was 150.7/93.2 $\pm 10.7 / 11.0 \mathrm{mmHg}$, the clinic BP was 150.2/91.4 $\pm 10.6 / 9.4 \mathrm{mmHg}$ and the clinic CASP was $136.3 \pm 12.2 \mathrm{mmHg}$.
At the end of the study, 59\% of the 44 patients were taking valsartan > OM $80 \mathrm{mg}$, while $41 \%$ were taking OM $80 \mathrm{mg}$. After 12 weeks of treatment, there were reductions in the clinic SBP $(-14.9 \pm 10.7 \mathrm{mmHg}, \mathrm{p}<0.001)$, DBP $(-10.9 \pm 8.4 \mathrm{mmHg}$, $\mathrm{p}<0.001)$ and CASP $(-15.3 \pm 10.9 \mathrm{mmHg}, \mathrm{p}<0.001$; Table II). About $57 \%$ of the treatment cohort achieved BP control (i.e. BP $<140 / 90 \mathrm{mmHg}$ ). Multiple linear regression showed that gender $(p=0.578)$; treatment history, i.e. on pre-existing treatment or treatment-naïve $(p=0.966)$; and final dose, i.e. dichotomised at $80 \mathrm{mg}$ or $>80 \mathrm{mg}$, ( $p=0.727)$ did not significantly affect CASP reduction. However, baseline SBP was found to have a significant impact $(p<0.001)$ on CASP reduction - after controlling for the aforementioned covariates, every one unit increase in baseline SBP resulted in a 0.6 -unit increase in CASP reduction. 
Table I. Baseline demographics of patients with uncontrolled hypertension* who were started on valsartan treatment $(n=44)$.

\begin{tabular}{lc}
\hline Variable & No. (\%) \\
\hline Age $^{+}(\mathbf{y r})$ & $35 \pm 16$ \\
Gender & \\
Male & $33(75)$ \\
Female & $11(25)$ \\
Body mass index ${ }^{+} \mathbf{( k g / \mathbf { m } ^ { 2 } )}$ & $27 \pm 8$ \\
Ethnicity & \\
Chinese & $35(80)$ \\
Malay & $5(11)$ \\
Indian & $3(7)$ \\
Others & $1(2)$ \\
Pre-existing treatment & \\
No. of drugs & \\
0 & $30(68)$ \\
1 & $9(20)$ \\
2 & $3(7)$ \\
3 & $2(5)$ \\
Type of drug & \\
ACE inhibitor & \\
Beta blocker & $3(7)$ \\
Calcium channel blocker & $17(39)$ \\
Diuretic & $1(2)$ \\
Diamorbidity & $2(5)$ \\
Hyperlipidaemia & $11(25)$ \\
Chronic renal impairment & $33(75)$ \\
Stroke & $3(7)$ \\
Ischaemic heart disease & \\
Yes & \\
No & \\
\hline
\end{tabular}

*Confirmed on 24-hour ambulatory blood pressure monitoring. †Data presented as mean \pm standard deviation. ACE: angiotensin-converting enzyme

Table II. Brachial and central blood pressure parameters after 12 weeks of treatment with valsartan $(n=44)$.

\begin{tabular}{|c|c|c|c|c|}
\hline \multirow[t]{2}{*}{ Variable } & \multicolumn{3}{|c|}{ Mean \pm standard deviation } & \multirow[t]{2}{*}{ p-value } \\
\hline & At baseline & $\begin{array}{c}\text { After } \\
12 \text { weeks }\end{array}$ & Change & \\
\hline $\begin{array}{l}\text { Clinic SBP } \\
(\mathrm{mmHg})\end{array}$ & $150.2 \pm 10.6$ & $135.3 \pm 9.3$ & $-14.9 \pm 10.7$ & $<0.001$ \\
\hline $\begin{array}{l}\text { Clinic DBP } \\
(\mathrm{mmHg})\end{array}$ & $91.4 \pm 9.5$ & $80.6 \pm 8.8$ & $-10.9 \pm 8.4$ & $<0.001$ \\
\hline CASP $(\mathrm{mmHg})$ & $136.6 \pm 12.3$ & $121.1 \pm 10.4$ & $-15.3 \pm 10.9$ & $<0.001$ \\
\hline $\begin{array}{l}\text { Brachial PP } \\
(\mathrm{mmHg})\end{array}$ & $57.8 \pm 12.7$ & $54.4 \pm 11.1$ & $-3.4 \pm 12.4$ & 0.078 \\
\hline $\begin{array}{l}\text { Central PP } \\
(\mathrm{mmHg})\end{array}$ & $45.2 \pm 11.6$ & $41.0 \pm 10.8$ & $-4.3 \pm 10.1$ & 0.009 \\
\hline PP ratio & $1.32 \pm 0.16$ & $1.36 \pm 0.18$ & $0.03 \pm 0.14$ & 0.110 \\
\hline
\end{tabular}

CASP: central aortic systolic pressure; DBP: diastolic blood pressure; PP: pulse pressure; SBP: systolic blood pressure

We also looked at the pulse pressure (PP) ratio (brachial PP divided by central PP), as it is a common parameter reported in other studies on central pressure. PP ratio is the degree of PP amplification as the pressure wave moves away from the heart (with the PP in the brachial artery being higher than that in the aorta). In the treatment cohort, there was a small increase in the PP ratio (from 1.32 to 1.36), although this was not statistically significant $(p=0.110)$.

Correlation analysis demonstrated a moderately strong correlation between brachial SBP and CASP reduction (Pearson correlation coefficient $0.84, p<0.001$ ). Scatter plots for patients who were on pre-existing treatment for hypertension and those who were newly diagnosed (i.e. treatment-naïve) depicted similar lines of best fit (Fig. 3). Overall, simple linear regression showed that for every $1.0-\mathrm{mmHg}$ reduction in brachial SBP, there was a corresponding $0.8-\mathrm{mmHg}$ drop in CASP. Using ROC curve analysis of the treatment cohort at Week 12 (Fig. 4), a cut-off of $122.5 \mathrm{mmHg}$ for CASP (area under curve: 0.90) was chosen, as it was found to discriminate between controlled and uncontrolled BP (i.e. BP $\geq 140 / 90 \mathrm{mmHg}$ ) with optimal sensitivity and specificity ( $74 \%$ and $88 \%$, respectively).

\section{DISCUSSION}

The present study demonstrated a mean reduction of $15.3 \mathrm{mmHg}$ in CASP and a corresponding SBP reduction of $14.9 \mathrm{mmHg}$ (measured using the BPro watch) in our Asian cohort, after treatment with valsartan for 12 weeks. This reduction was comparable to that seen in studies conducted in Western populations using the SphygmoCor device; Klingbeil et al reported a 12.9-mmHg reduction in CASP (and a corresponding 13.5-mmHg reduction in SBP) after six weeks of valsartan $80 \mathrm{mg}$ monotherapy, ${ }^{(5)}$ while Boutouyrie et al reported a $13.7-\mathrm{mmHg}$ reduction in CASP (and a corresponding 12.9-mmHg reduction in SBP) after 24 weeks of additional amlodipine/valsartan 5/160 mg combination therapy. ${ }^{(6)} \mathrm{A}$ few midterm studies using other ARBs and the SphygmoCor device also showed the positive effects of ARBs on CASP - monotherapy with eprosartan $600 \mathrm{mg}$ daily for six weeks resulted in a 16- $\mathrm{mmHg}$ reduction in both SBP and CASP, ${ }^{(7)}$ while irbesartan-based therapy for six months resulted in a $12-\mathrm{mmHg}$ reduction in both SBP and CASP. ${ }^{(8)}$

In previous studies, PP amplification has been used to determine central haemodynamic effects. ${ }^{(4)} \mathrm{A}$ higher PP ratio suggests a more favourable central response. There was a 0.04 increase in the PP ratio at the end of the present study, which was similar to that seen in the EXPLOR study (i.e. 0.05 increase). ${ }^{(6)}$ Similarly, in another study where eprosartan was the treatment drug used, an increase of 0.04 in the PP ratio was reported. ${ }^{(7)}$

In the present study, correlation analysis showed a moderate correlation between brachial SBP and CASP reductions, while linear regression analysis revealed that for every $1.0-\mathrm{mmHg}$ reduction in brachial SBP, the CASP reduction was $<1.0 \mathrm{mmHg}$. This finding contrasted with the result obtained via paired $t$-test, which showed that mean CASP reduction was similar to mean SBP reduction. We believe that the results of the correlation analysis is more reflective of the haemodynamic response, as each data point linked both the SBP and CASP reductions in the same patient, unlike the paired $t$-test, which compared only the 


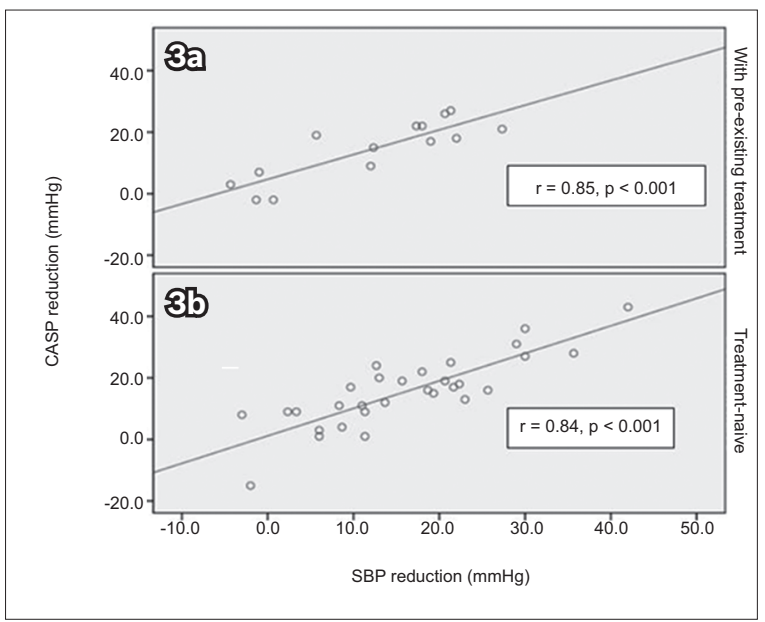

Fig. 3 Scatter plots for patients who were (a) on pre-existing treatment for hypertension; and (b) newly diagnosed (i.e. treatment-naïve) show similar lines of best fit. The lines of best fit show the relationship between centra aortic systolic pressure (CASP) reduction and systolic blood pressure (SBP) reduction in patients treated with valsartan.

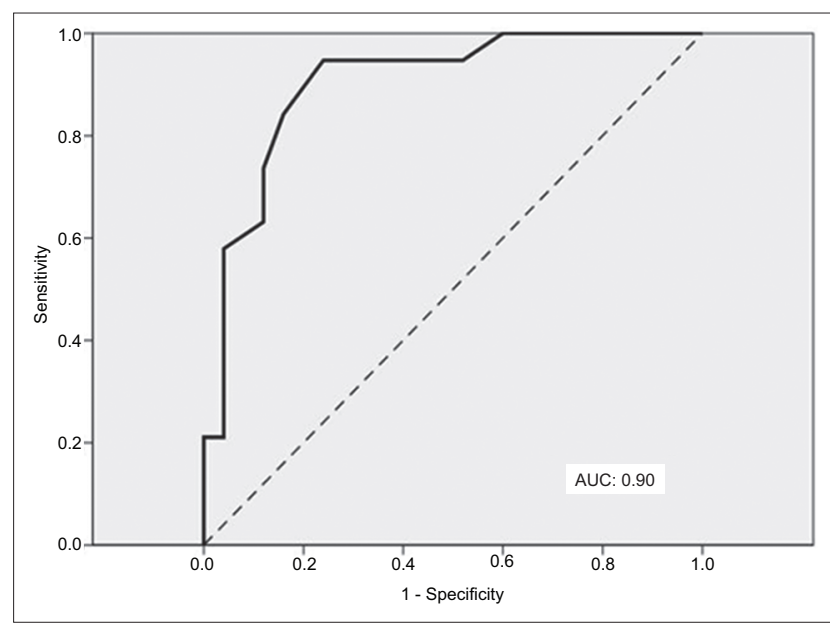

Fig. 4 Receiver operating characteristic curve using central aortic systolic pressure to define blood pressure control shows area under curve (AUC) of 0.90 (excellent accuracy).

mean CASP and SBP reductions of the cohort. More correlation studies using other drugs would be useful to determine whether this type of analysis is better for comparing the ability of different drugs in lowering CASP beyond brachial SBP lowering than the usual reporting using mean CASP reduction or $\mathrm{PP}$ ratio.

One barrier to the use of CASP in titrating BP medication is the lack of guidelines regarding the optimal CASP target control. While the results of the present study suggest that, for a target treatment brachial BP control of $<140 / 90 \mathrm{mmHg}$, the equivalent CASP threshold might be around $\leq 123 \mathrm{mmHg}$, more studies are needed to determine the optimal CASP for the general hypertensive population. Of note, a recent Japanese study reported a baseline CASP of $122 \mathrm{mmHg}$ in a cohort of normotensive persons who eventually developed hypertension after three years of follow-up. ${ }^{(14)}$ Data from the Anglo-Cardiff Collaborative Trial II study suggested that the CASP cut-off value might approximate $125 \mathrm{mmHg}$; this value was 'translated' from the usual 140/90 $\mathrm{mmHg}$ brachial SBP cut-off for healthy and treatment-naïve hypertensive persons. ${ }^{(3)}$
We acknowledge that there was a lack of a comparative or control arm in the present study; we demonstrated only the pre- and post-effect of valsartan treatment on CASP. This weakness, which would have led to a regression artefact, could have resulted in overestimation of the treatment effect of valsartan. In our study, the brachial BP control rate of the treatment cohort was about $57 \%$; in other controlled studies with valsartan-based treatments, the control rate was reported to range from $47 \%$ to $61 \%{ }^{(6,15)}$ Furthermore, as mentioned in the previous paragraphs, the CASP reduction in our study was comparable to that of other controlled studies. Thus, we do not think that the lack of a control arm significantly affected the results of our study.

Another limitation of the present study was the heterogeneity of the treatment cohort. Firstly, no washout was done for the group that had pre-existing treatment (32\% of the treatment cohort). Thus, the results observed could have been due to interactions between the baseline drugs and valsartan, and not just valsartan alone. Secondly, the valsartan dose was titrated at Week 4 to obtain the final dose, which was used over the last eight weeks of the treatment phase. This would have resulted in variable dosing within and among the patients in the treatment cohort over the 12 weeks of treatment. Although forced titration and fixed dosing are the norm in conventional drug studies, we deviated from the norm so as to ensure patient safety and, thus, promote patient participation. In fact, the present study was conducted in a manner that reflects actual clinical practice, in which a clinician might add on a new medication to a patient's existing regimen at a dose that is deemed best for the patient, based on the patient's risk profile and acceptance level, with dose titration performed when needed. Despite the heterogeneity of the treatment cohort, final drug dosage and pre-existing treatment were not shown to significantly influence the mean CASP reduction on multivariate analysis.

As the mean age of our study cohort was relatively young (i.e. 35 years), caution should be exercised when extrapolating the results of the present study to other populations, especially elderly populations who may have arterial stiffness, yet are known to be less responsive to ACE-I and ARB treatments.

While the SBP and CASP reductions after valsartan treatment were quite similar in the present study, it should not be presumed that when a different drug or drug combination is used, the extent of reduction for SBP and CASP would also be similar. If advances in BP measurement technology can lead to the ability to directly monitor CASP, even in the primary setting, then concerns about the differential effects of drugs on central and peripheral BP would cease to be an issue. However, until then, there is a need for further studies comparing the relationship between brachial and central BP reductions using different drugs, in order to guide the use of antihypertensive drugs. Outcome studies based on central pressure titration are also needed.

To conclude, using radial tonometry, we demonstrated good correlation between CASP and brachial SBP reductions after 12 weeks of treatment with valsartan in a relatively young hypertensive Asian cohort. Correlation between CASP and SBP reductions may be used to indicate the ability of a drug to 
lower CASP beyond SBP lowering. More studies are required to determine the optimal CASP target for the general population.

\section{ACKNOWLEDGEMENTS}

We would like to thank Dr Pankaj Handa and Dr Veerendra Chadachan from the Vascular Unit of Tan Tock Seng Hospital, Singapore, for their contributions toward the design of this study. We also thank the founder of HealthSTATS Int' Pte Ltd, Dr Ting Choon Meng, who provided invaluable advice on the use of the BPro watch and software programs. Our deepest gratitude also goes to the staff of the Clinical Research Unit of Tan Tock Seng Hospital, Singapore, especially Dr Leong Khai Pang, Ms Noriza Mustafa, and the statisticians (Mr Chua Kia Chong, Mr Wilson Low, Ms Mira Shen) and study coordinators for their immense support. We would like to acknowledge Novartis (Singapore) Pte Ltd for sponsoring the valsartan drug, BPro watches and A-Pulse CASP program for this investigator-initiated study.

\section{REFERENCES}

1. Williams B, Lacy PS, Thom SM, et al; CAFE Investigators; AngloScandinavian Cardiac Outcomes Trial Investigators; CAFE Steering Committee and Writing Committee. Differential impact of blood pressurelowering drugs on central aortic pressure and clinical outcomes: principal results of the Conduit Artery Function Evaluation (CAFE) study. Circulation 2006; 113:1213-25.

2. Camacho F, Avolio A, Lovell NH. Estimation of pressure pulse amplification between aorta and brachial artery using stepwise multiple regression models. Physiol Meas 2004; 25:879-89.

3. McEniery CM, Yasmin, McDonnell B, et al; Anglo-Cardiff Collaborative Trial Investigators. Central pressure: variability and impact of cardiovascular risk factors: the Anglo-Cardiff Collaborative Trial II. Hypertension 2008; 51:1476-82.

4. Protogerou AD, Stergiou GS, Vlachopoulos C, Blacher J, Achimastos A.
The effect of antihypertensive drugs on central blood pressure beyond peripheral blood pressure. Part II: Evidence for specific class-effects of antihypertensive drugs on pressure amplification. Curr Pharm Des 2009; 15:272-89.

5. Klingbeil AU, John S, Schneider MP, et al. AT1-receptor blockade improves augmentation index: a double-blind, randomized, controlled study. J Hypertens 2002; 20:2423-8.

6. Boutouyrie P, Achouba A, Trunet P, Laurent S; EXPLOR Trialist Group. Amlodipine-valsartan combination decreases central systolic blood pressure more effectively than the amlodipine-atenolol combination: the EXPLOR Study. Hypertension 2010; 55:1314-22.

7. Dhakam Z, McEniery CM, Yasmin, et al. Atenolol and eprosartan: differential effects on central blood pressure and aortic pulse wave velocity. Am J Hypertens 2006; 19:214-9.

8. Schneider MP, Delles C, Klingbeil AU, et al. Effect of angiotensin receptor blockade on central haemodynamics in essential hypertension: results of a randomised trial. J Renin Angiotensin Aldosterone Syst 2008; 9:49-56.

9. Williams B, Lacy PS, Yan P, et al. Development and validation of a novel method to derive central aortic systolic pressure from the radial pressure waveform using an n-point moving average method. J Am Coll Cardiol 2011; 57:951-61.

10. Theilade S, Hansen TW, Joergensen C, Lajer M, Rossing P. Tonometric devices for central aortic systolic pressure measurements in patients with type 1 diabetes: comparison of the BPro and SphygmoCor devices. Blood Press Monit 2013; 18:156-60.

11. Sule AA, Teong HH, Tay JC. Very high central aortic systolic pressures in a young hypertensive patient on telmisartan: Is central aortic systolic pressure associated with white coat hypertension? Int J Angiol 2010; 19:e132-4.

12. Nair D, Tan SY, Gan HW, et al. The use of ambulatory tonometric radial arterial wave capture to measure ambulatory blood pressure: the validation of a novel wrist-bound device in adults. J Hum Hypertens 2008; 22:220-2.

13. Faul F, Erdfelder E, Lang AG, Buchner A. G*Power 3: a flexible statistical power analysis program for the social, behavioral, and biomedical sciences. Behav Res Methods 2007; 39:175-91.

14. Tomiyama $\mathrm{H}, \mathrm{O}^{\prime}$ rourke MF, Hashimoto $\mathrm{H}$, et al. Central blood pressure: a powerful predictor of the development of hypertension. Hypertens Res 2013; 36:19-24.

15. Julius S, Kjeldsen SE, Brunner $H$, et al. VALUE trial: Long-term blood pressure trends in 13,449 patients with hypertension and high cardiovascular risk. Am J Hypertens 2003; 16:544-8. 